\title{
The effect of sex and age on cortical grey and white matter volumes of frontal lobe
}

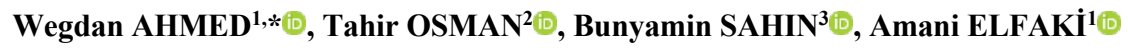 \\ ${ }^{1}$ Department of Anatomy, Faculty of Medicine, National University, Khartoum, Sudan \\ ${ }^{2}$ Department of Anatomy, Faculty of Medicine, Ondokuz Mayis University, Samsun, Turkey \\ ${ }^{3}$ Faculty of Graduate Studies and Scientific Research, The National Ribat University, Khartoum, Sudan
}

\begin{abstract}
\begin{tabular}{ccccc}
\hline Received: 27.07 .2020 & $\bullet$ & Accepted/Published Online: 24.02 .2021 & Final Version: 23.04 .2021 \\
\hline
\end{tabular}
\section{Abstract}

Grey matter (GM) of the frontal lobe carries out its complex function such as muscle control, speech, decision making, and self-control; while the white matter (WM) of frontal lobe is a major contributor to human brain enlargement and higher structural connectivity. This study was conducted to assess the effect of sex and age on grey and white matter volumes (GMV and WMV) of the frontal lobe and its gyri in healthy young adult Sudanese. The present study included 139 healthy Sudanese subjects ( 80 males and 59 females). Participant's ages were ranging between $20-40$ years. T1-weighted MR brain images with thickness $1 \mathrm{~mm}$ were obtained. MR images of the subjects were analyzed using the automatic segmentation software (BrainSuite). GMV and WMV of frontal lobe and its gyri were estimated using the output data of the process of software. Males had greater GMV and WMV of the frontal lobes $\left(251.18 \pm 31.80 \mathrm{~cm}^{3}\right.$ and $143.48 \pm 22.92 \mathrm{~cm}^{3}$, respectively) and most of frontal lobe gyri $(\mathrm{P}<0.05)$. Change with age in GMV has been reported in the frontal lobes, superior frontal gyrus, pars orbitalis, orbitofrontal, and cingulate gyrus $(\mathrm{P}<0.05)$. Change with age in WM volumes has been found in the cingulate, precentral, and paracentral gyri $(\mathrm{P}<0.05)$. Sex has prominent effect on GMV and WMV of the frontal lobes and most of frontal lobe gyri, thus sex is a major contributor to GMV and WMV differences between individuals. Age effect GMV and WMV of most frontal lobe gyri, these findings confirm the continuation of maturation of frontal lobe until fourth decade. Assessing effect of sex and age on GM and WM in healthy adult has major importance to distinguish the normal brain from diseased.
\end{abstract}

Keywords: grey matter volume, white matter volume, BrainSuite

\section{Introduction}

Frontal lobe occupies an exalted position in neuroscience and become one of the most important area of research in human brain due to its functional characteristics. Frontal lobe is responsible for planning and execution of the movement, formation of words, problem solving, decision making, cognitive flexibility, and working memory (Elaine N. Marieb, 2007; Welsh et al., 1991).

The frontal lobe parenchyma consists mainly of grey matter (GM) and white matter (WM), that differ in the structural and functional. GM consists mainly of nerve cell bodies; where almost all interactions between neurons take place and processes the complex functioning of the frontal lobe (Miller, 1980). WM composes mainly of nerve fibers (Snell, 2010); which proposes as a major contributor to human brain enlargement and higher structural connectivity (Schoenemann et al., 2005). On the basis of MR image, the brain tissue can be classified into GM, WM, and CSF (cerebro-spinal fluid). This classification can be done by using the manual segmentation, but it is hard, time consuming, and subjective (Kasiri et al., 2010). Therefore, there are strong demands to perform the reliable and accurate automated segmentation of brain MR images as a prerequisite for the comprehensive brain analysis (Kasiri et al., 2010). In this study, an automated segmentation software called Brain-Suite was used, it provides a comprehensive approach to extracting a surface multi representation of the cerebral cortex from T1-weighted MR images and segments and labels grey and white matter structures. Although, the effect of sex and age on grey matter has been widely studied; the results regarding young adults were not analyzed separately and mixed with other age groups (younger or older). Therefore, the variations observed could not be applied specifically to individuals in the second and third decade.

In the present study, the grey and white matter volumes of the frontal lobes and their gyri were measured from T1weighted MR brain images by applying automatic segmentation software (Brain-Suite); to assess effect of sex and age in cortical grey and white matter volumes of the frontal lobe and its gyri in sample of 139 healthy young adult Sudanese ranging from age 20 to 40 years. 


\section{Materials and methods}

\subsection{Subjects}

The current study included 139 healthy young adult Sudanese subjects; age of participants was ranging between 20-40 years. Sexes were match in their age (age of males and females were $28 \pm 5.72$ and $28 \pm 6.00$ years, respectively) and body mass index (BMI) (BMI of males and females were $23.93 \pm 3.6$ and $24.89 \pm 5.07 \mathrm{~kg} / \mathrm{cm}^{2}$, respectively).

The participants were excluded if they are drug abuse; had head trauma, neurological diseases, psychiatric diseases, and congenital malformation related to the brain. The study was approved by the Ethical Committee of the National Ribat University.

\subsection{Magnetic resonance imaging}

Structural MRI was done in radiology department, Doctors' Clinic. MR imaging was performed on 1.5 Tesla Philips scanner, Version: 3.2.1. T1-weighted MR brain images obtained using three-dimensional acquisition by Magnetization Prepared Rapid Acquisition (MP-RAGE), which produces good grey/white matter contrast in coronal section, acquisition time ( 5 minutes and 18 seconds), slice distance is $1.0 \mathrm{~mm}$, the field of view is $250 \mathrm{read}, 192 \mathrm{~mm}$ phase, $\mathrm{TR}=1657 \mathrm{~ms}$, $\mathrm{TE}=2.95 \mathrm{~ms}$, bandwidth $180 \mathrm{~Hz} /$ pixel, flip angle $15^{\circ}$, ECHO spacing $=7.5 \mathrm{~ms}$, phase resolution $=100 \%$, and slice resolution $=50 \%$.

\subsection{MR images analysis}

MR images of the subjects were analyzed using automatic segmentation software (BrainSuite 13a). BrainSuite is "a collection of software tools that enable largely automated processing of magnetic resonance images (MRI) of the human brain". BrainSuite software was performed on a Toshiba computer Core i3, $2.10 \mathrm{GHz}, 6 \mathrm{~GB}$. The software analyzed each MR image in two stages: the first stage is cortical surface extraction sequence, which take 30 minutes to run; while the second stage two is long and last two hours and 15 minute, which is surface and volume registration.

\subsection{Cortical surface extraction sequence (CSE)}

The first step in the CSE is the skull stripping, which include removing of the skull and scalp from MR image. After completion of this stage, be sure that the edge detection line surrounded only the brain tissue, if not you have to adjust it by changing the diffusion iterations, diffusion constant, and edge constant from the skull stripping dialog. The next step is nonuniformity correction, which corrects the skull stripped MR image from shading artifacts. These shading artifacts can produced by imperfections in the field coils used in the systems. Then, tissue classification and cerebrum Labelling, during these steps, the software classifies the tissue into cerebrospinal fluid (CSF), grey and white matter; and labelling the brain volume into cerebrum, cerebellum, and brainstem. After that the BrainSuite running Cortex Masks Selection and topology correction that include extraction of the cerebral cortex and correction of any errors that may occurs during this process. The last stages in the cortical surface extraction include the generation of the pial surface and separation of the cortical surface into left and right hemispheres and displays them with different color (Fig. 1).

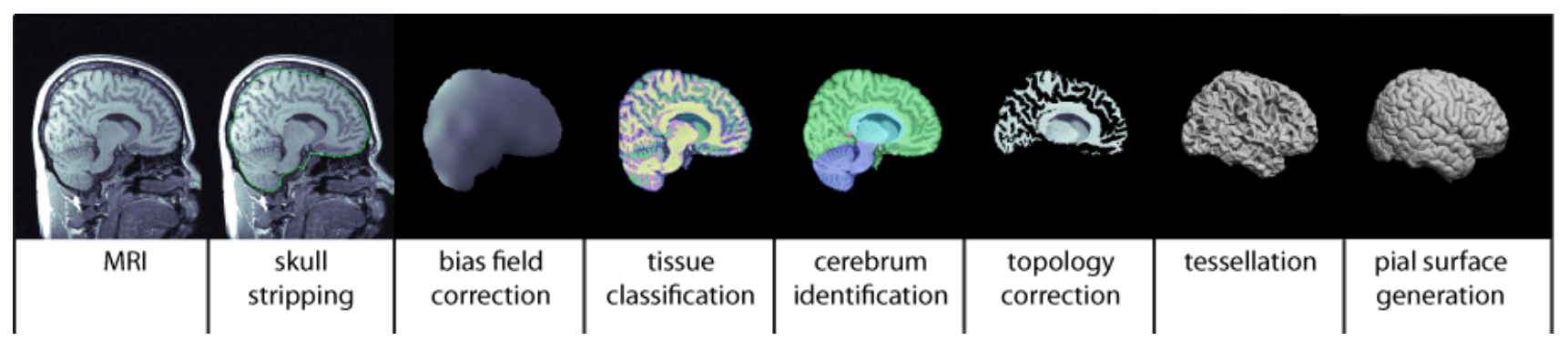

Fig. 1. Cortical surface extraction sequence

\subsection{Surface and volume registration (SVReg)}

Registering images to a common space also allows for automatic labeling of cortical and subcortical structures, allowing for analysis of ROIs to be automated. BrainSuite "comes with SVReg, a program that registers the surfaces and volumes generated by the Cortical Surface Extraction Sequence to a brain atlas manually labeled by an expert neuroanatomist". Stages of SVReg: Surface Smoothing and Coarse Alignment of Smoothed Surfaces, during these stages the software smoothing the cortical surfaces mesh and producing alignment of the cortex; then aligning these smoothed surfaces in 3D space. After that is atlas to subject registration, this stage needs for Curvature-Based Alignment, which is applying of sulcal curves and cortical labels from the atlas to the subject's cortical surface. The next steps are Refinement of the labels and sulcal curves and Harmonic
Extension, the software performed volumetric spatial alignment of brains by extending the surface registration to the entire volume. Finally, is Elastic Deformation, the output of this process is a point correspondence between the two brain volumes based on the deformation required to map one to the other. Once the atlas volume is registered to the subject's volume, the atlas' warped label volume acts as a label volume for the subject .

The results of SVReg were labelled inner, pial and mid cortical surfaces of cerebrum and spread sheet of statistic include measurement of the cortical and subcortical structures (Fig. 2). BrainSuite automatically calculate cortical structures of gyri of the cerebral hemispheres. To calculate cortical structures of cerebral hemisphere the following formulas (1-5) were used in Microsoft excel worksheet. 
Ahmed et al. / J Exp Clin Med

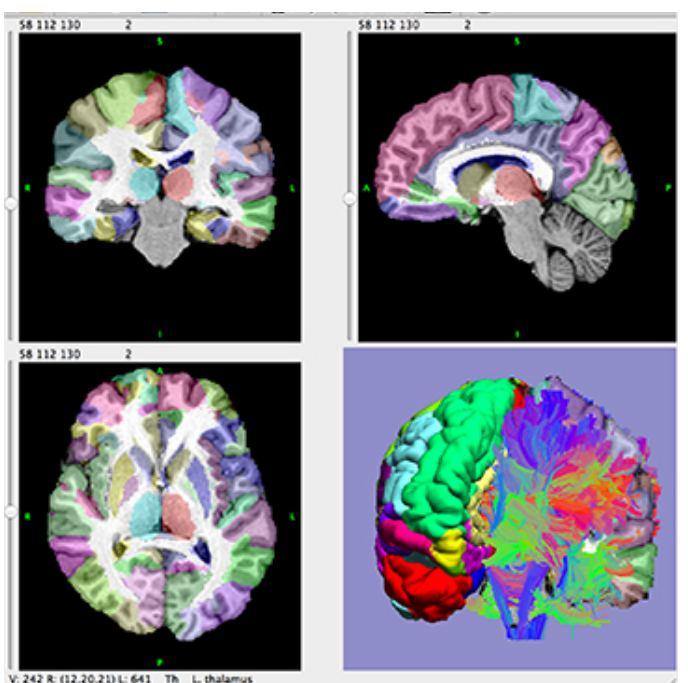

Fig. 2. MR image analyzed by BrainSuite

\subsection{Statistical analysis}

Data were analyzed using Statistical Package of Social Science (SPSS) version 21.0. Independent sample t. test was performed to compare mean values of grey and white matter between males and female. Bivariate correlation was used to evaluate the relationship of grey and white matter with age. $\mathrm{P}$ value equal or less than 0.05 was considered statistically different.

\section{Results}

\subsection{Sex differences in grey matter volume (GMV)}

Quantitative data of GMV of the frontal lobes and frontal lobe gyri are shown in Table 1. Males had greater GMV in the right, left, and total frontal lobes; all right frontal lobe gyri; and left and total middle frontal gyri, pars orbitalis, and cingulate gyri; the total precentral and transverse frontal gyri $(\mathrm{P}<0.05)$. Conversely, there were no differences between genders in GMV of the other frontal lobe gyri $(\mathrm{P}>0.05)$ (Table 1).

Table 1. Grey matter volumes of frontal lobes and frontal lobe gyri

\begin{tabular}{|c|c|c|c|c|c|c|}
\hline \multirow{3}{*}{ Frontal lobe Gyri } & \multicolumn{6}{|c|}{ Grey Matter Volume $\left(\mathrm{cm}^{3}\right)$} \\
\hline & \multicolumn{2}{|c|}{ Right } & \multicolumn{2}{|c|}{ Left } & \multicolumn{2}{|c|}{ Total } \\
\hline & Males & Females & Males & Females & Males & Females \\
\hline Frontal Lobe & $132.04 \pm 15.9$ & $117.23 \pm 11.73 *$ & $131.79 \pm 15.7$ & $116.81 \pm 11.31 *$ & $263.82 \pm 31.60$ & $234.05 \pm 22.95^{*}$ \\
\hline Superior Frontal Gyrus & $38.54 \pm 4.96$ & $34.10 \pm 3.72 *$ & $38.43 \pm 5.55$ & $37.17 \pm 4.70$ & $75.74 \pm 10.36$ & $72.94 \pm 9.18$ \\
\hline Middle Frontal Gyrus & $19.08 \pm 3.70$ & $16.74 \pm 2.96^{*}$ & $19.73 \pm 3.62$ & $17.92 \pm 2.99 *$ & $38.20 \pm 7.34$ & $35.50 \pm 5.43^{*}$ \\
\hline Pars Opercularis & $4.99 \pm 0.90$ & $4.46 \pm 0.89^{*}$ & $4.66 \pm 0.88$ & $4.57 \pm 0.83$ & $9.53 \pm 1.63$ & $9.19 \pm 1.51$ \\
\hline Pars Triangularis & $9.11 \pm 2.17$ & $7.96 \pm 1.68^{*}$ & $6.93 \pm 1.30$ & $6.79 \pm 1.18$ & $15.69 \pm 3.07$ & $15.24 \pm 2.623$ \\
\hline Pars Orbitalis & $1.84 \pm 0.36$ & $1.67 \pm 0.36^{*}$ & $2.50 \pm 0.35$ & $2.30 \pm 0.34^{*}$ & $4.29 \pm 0.64$ & $4.02 \pm 0.56^{*}$ \\
\hline Precentral Gyrus & $14.79 \pm 2.38$ & $13.59 \pm 1.70^{*}$ & $14.07 \pm 2.29$ & $13.46 \pm 1.92$ & $28.71 \pm 4.32$ & $27.26 \pm 3.49^{*}$ \\
\hline Paracentral gyrus & $4.78 \pm 0.77$ & $4.15 \pm 0.56^{*}$ & $4.22 \pm 0.72$ & $4.08 \pm 0.59$ & $8.79 \pm 1.45$ & $8.51 \pm 1.11$ \\
\hline Transverse Frontal Gyrus & $3.14 \pm 0.55$ & $2.79 \pm 0.61^{*}$ & $2.73 \pm 0.55$ & $2.56 \pm 0.51$ & $5.85 \pm 1.02$ & $5.39 \pm 0.90^{*}$ \\
\hline Cingulate Gyrus & $15.81 \pm 2.24$ & $13.88 \pm 1.76^{*}$ & $16.53 \pm 2.65$ & $15.64 \pm 2.26^{*}$ & $31.82 \pm 4.91$ & $30.23 \pm 4.25^{*}$ \\
\hline Subcallosal Area & $0.32 \pm 0.08$ & $0.29 \pm 0.07 *$ & $0.34 \pm 0.09$ & $0.34 \pm 0.09$ & $0.65 \pm 0.15$ & $0.64 \pm 0.13$ \\
\hline Orbito-Frontal Gyri & $19.62 \pm 2.41$ & $17.23 \pm 1.80^{*}$ & $17.65 \pm 2.40$ & $16.97 \pm 2.19$ & $36.51 \pm 4.82$ & $35.23 \pm 4.43$ \\
\hline
\end{tabular}

\subsection{Sex differences in white matter volume}

Quantitative data of WMV of frontal lobes and frontal lobe gyri are shown in table 2. Males had greater WMV in the right, left, and total frontal lobes; all right frontal lobe except pars orbitalis and subcallosal area; the left and total precentral gyri; the left middle frontal and cingulate gyri and pars orbitalis $(\mathrm{P}<0.05)$. Conversely, there were no differences between genders in WMV of the other frontal lobe gyri $(\mathrm{p}>0.05)$.

\subsection{Correlation between age and grey matter volume}

There was negative correlation between age and GMV of the right, left and total frontal lobes; the right superior frontal gyrus in males and females; the right pars orbitalis and orbitofrontal gyri in males; and the right cingulate gyrus in females (Figs. 410). However, there was no correlation between age and GMV of the right, left, and total pars opercularis, pars triangularis, middle frontal, precentral, paracentral, transverse frontal, and subcallosal area $(\mathrm{P}>0.05)$.

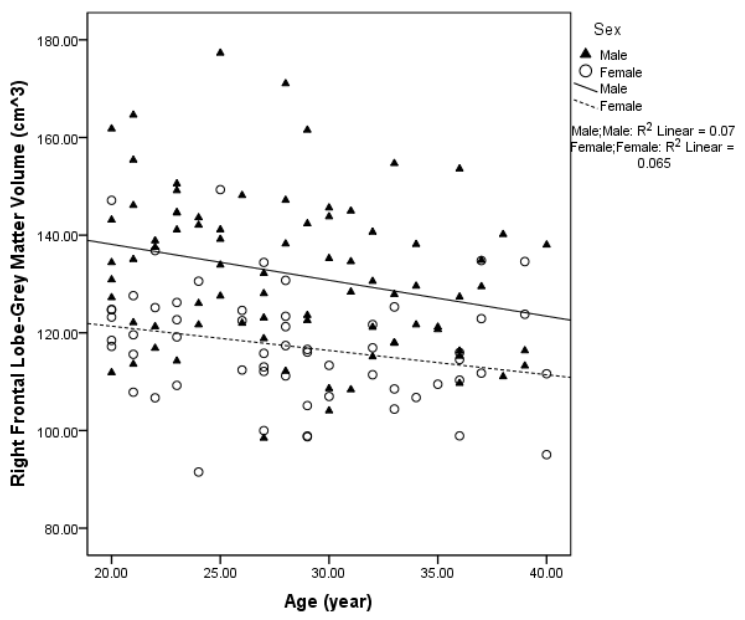

Fig. 3. Correlation between age and grey matter volume of right (RT) frontal lob 


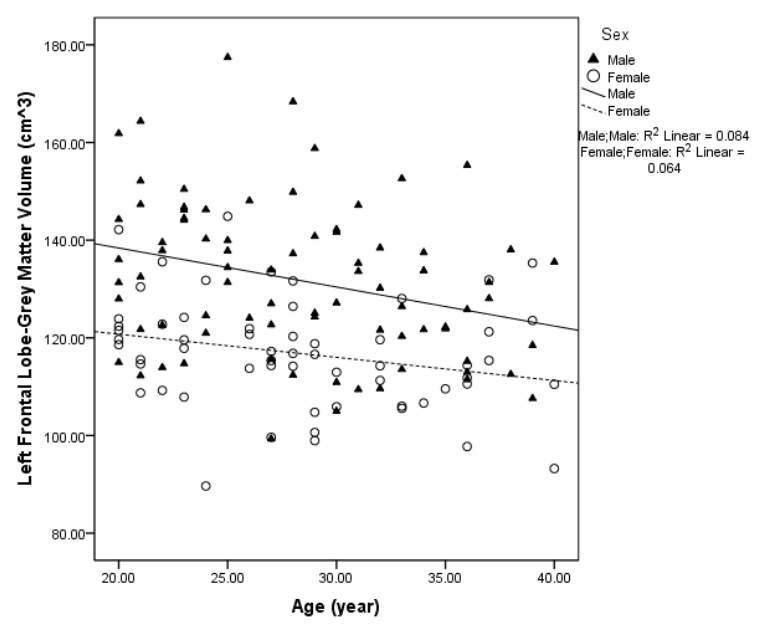

Fig. 4. Correlation between age and grey matter volume of left (LT) frontal lobe

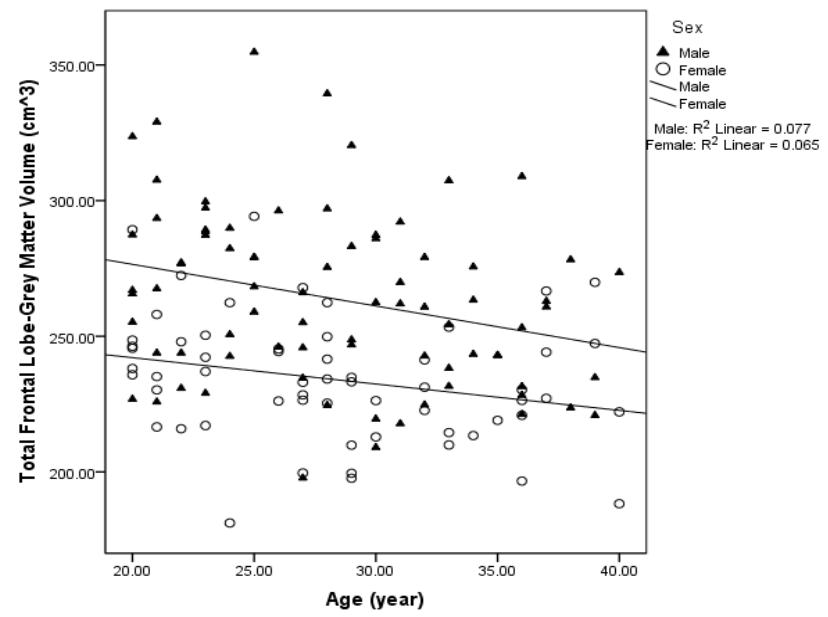

Fig. 5. Correlation between age and grey matter volume of total frontal lobe

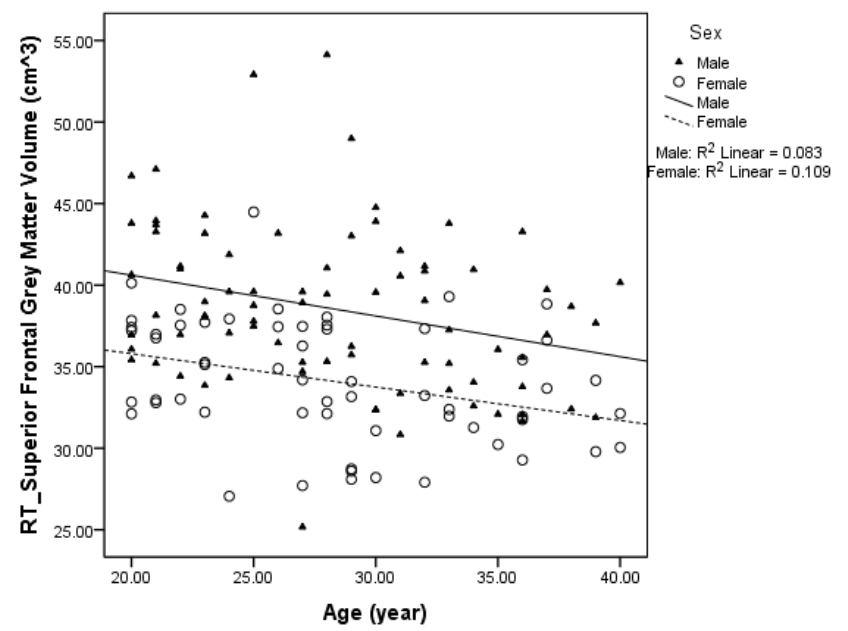

Fig. 6. Correlation between age and grey matter volume of right (RT) superior frontal gyrus

\subsection{Correlation between age and white matter volume}

There was positive correlation between age and WMV of the left and total cingulate gyri in females and right paracentral gyrus in males; but a negative correlation between age and WMV of the total precentral gyri in males ( $\mathrm{P}<0.05$, Figs. 1013). However, there was no correlation between age and WMV

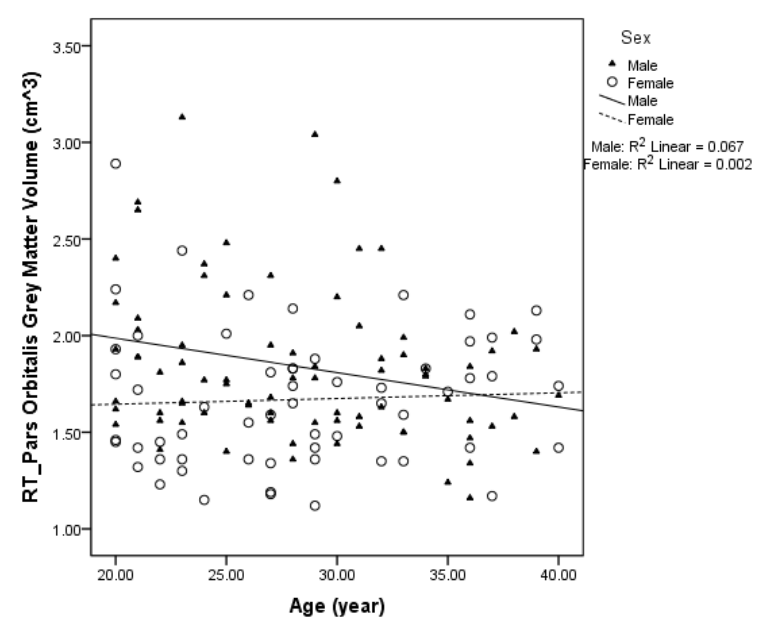

Fig. 7. Correlation between age and grey matter volume of right (RT) pars orbitalis

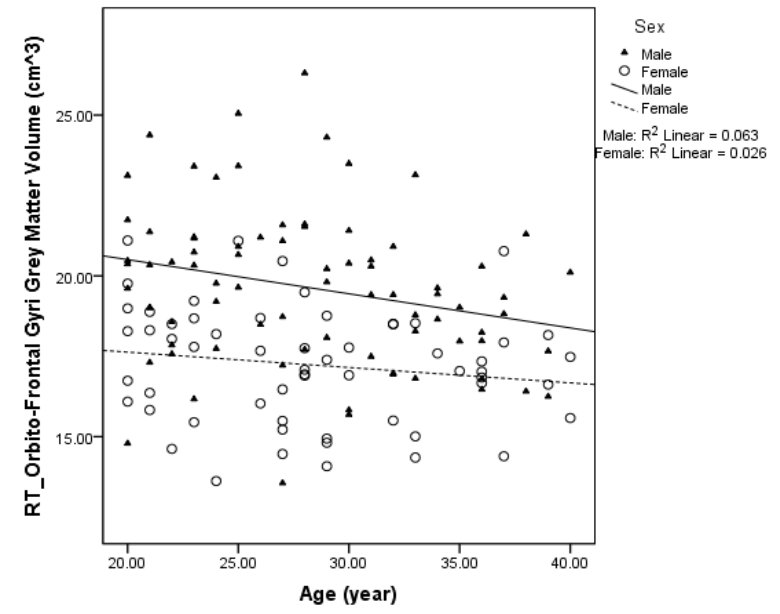

Fig. 8. Correlation between age and grey matter volume of right (RT) orbito-frontal gyri

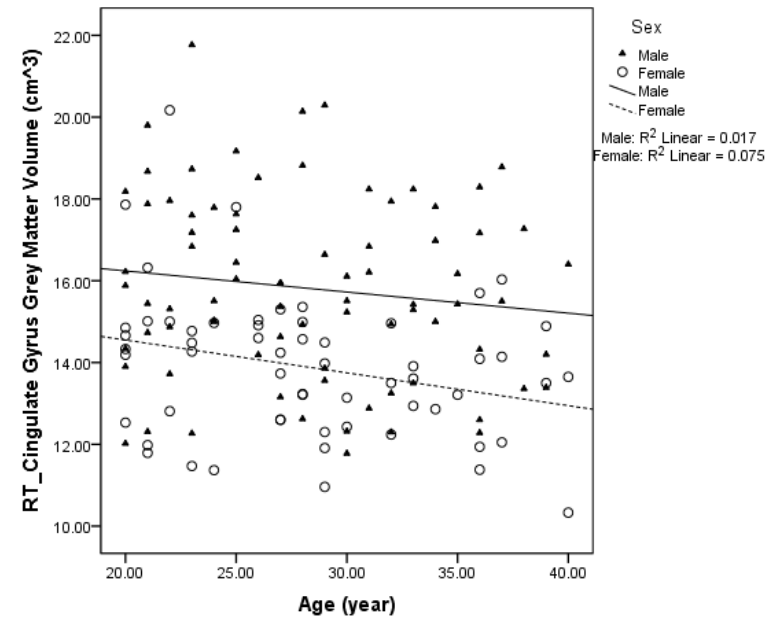

Fig. 9. Correlation between age and grey matter volume of right (RT) cingulate gyrus

of the right, left, and total superior frontal gyri, middle frontal gyri, pars opercularis, pars triangularis, pars orbitalis, transverse frontal gyri, orbito-frontal gyri, and subcallosal areas in males and females $(\mathrm{P}>0.05)$. 
Ahmed et al. / J Exp Clin Med

Table 2. White matter volumes of frontal lobes and frontal lobe gyri

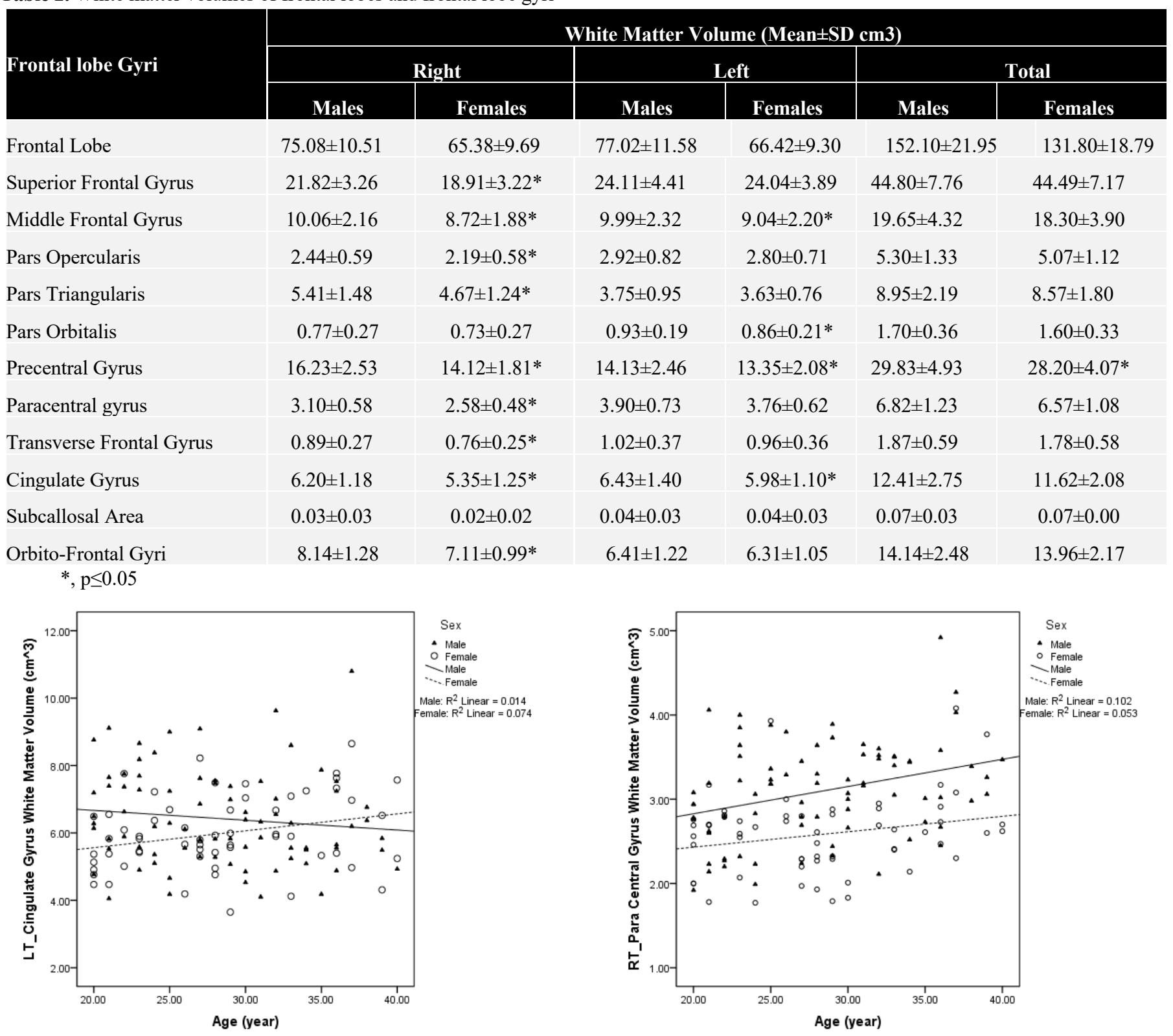

Fig. 10. Correlation between age and white matter volume of left (LT) cingulate gyrus

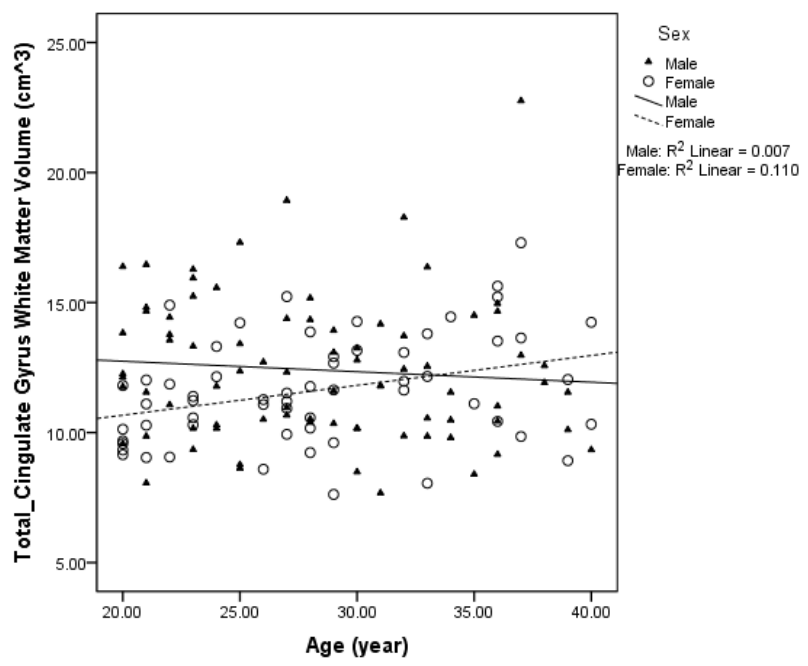

Fig. 11. Correlation between age and white matter volume of total cingulate gyri

Fig. 12. Correlation between age and white matter volume of right (RT) paracentral gyrus

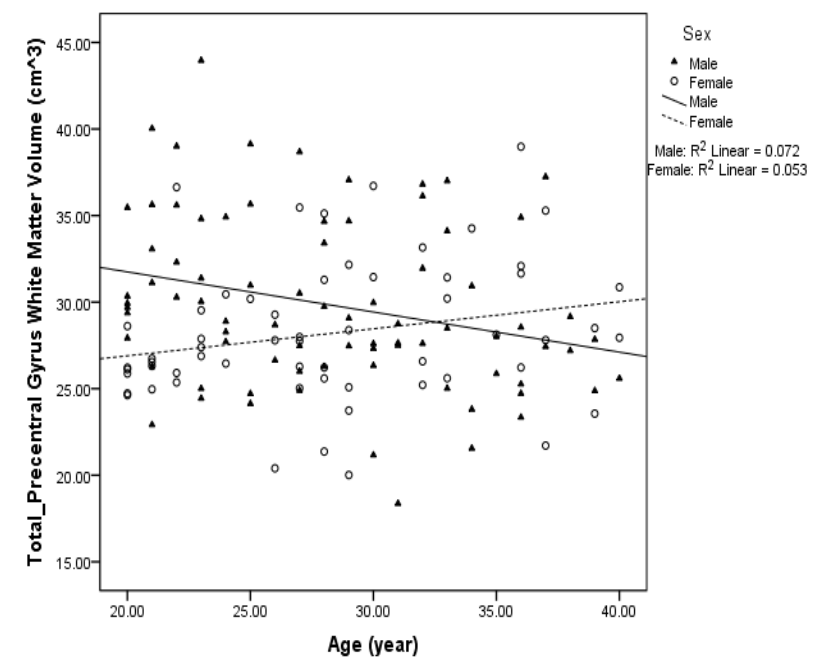

Fig. 13. Correlation between age and white matter volume of total precentral gyri 


\section{Discussion}

The present study provided a detailed description of the effect of sex and age on grey and white matter volumes of frontal lobe and its gyri. Firstly, it was compared mean values of grey and white matter volumes between males and females, to determine sex differences. Secondly, it was correlated grey and white matters volumes to age; to find out the correlations.

According to findings of the presents study, males had significantly greater GMV in the frontal lobe and most of the frontal lobe gyri. Our result in agree with other studies that found greater GMV in males in the left cingulate (Allen et al., 2003), right precentral, middle frontal, and transverse frontal gyri (Brabera et al., 2013). In contrast, other studies found greater GMV in males in the left pars opercularis (Witte et al., 2010); and in females in the left pars triangularis (Witte et al., 2010), right paracentral gyrus, right inferior frontal gyrus, and right and left cingulate gyri (Brabera et al., 2013), precentral gyrus and orbitofrontal cortex. The reasons for these diverging findings may be related to differences in sample size, composition of examined brains, methods to quantify the compartmental volumes, or lack of neuropsychological control.

The possible reasons for the sex differences in grey matter volume (GMV) is the influence of sex hormones. The recent study found a connection between GMV and hormone levels of testosterone, estrogen or progesterone (Witte et al., 2010); but it hasn't yet cleared the effect of these hormones in the brain(Van Amelsvoort et al., 2001).

Sex differences in the regional GMV might develop as a result of differential environmental and social influences. Interestingly, use-dependent influences like lifelong intensive training in specific cognitive or motor skills have been suggested to modulate regional GMV (Draganski et al., 2004).

Our results revealed that males had significantly greater WMV in the frontal lobes and most of frontal lobe gyri. Literatures of cortical white matter volumes (WMV) are not widely available; WMV was measured by other method rather than MR imaging such as diffusion tensor imaging. Our result in agree with other study found males had greater white matter volume in the lateral prefrontal cortex (Kennedy et al., 2009).

The white matter volume (WMV) is determined by the number of axons, their caliber and the thickness of myelin sheath produced by the oligodendrocytes (Paus and Toro, 2009). WM connect various grey matter areas of the brain to each other, and carry nerve impulses between neurons. Increase WMV in males compared to females probably means that more connections among neurons. The increase in WMV might also mean greater communication between the frontal lobe gyri and other parts of the brain (Schoenemann et al., 2005).

In the brain there are many receptors for sex hormones, which affect the neurodevelopment. The hormonal effects on the white matter development have been discussed based on findings from animal studies, which show that testosterone is associated with myelogenesis (Martini and Melcangi, 1991) and estradiol effects synaptogenesis and reduces the rate of myelination (Woolley et al., 1996).

An interesting result was sex differences in GMV and WMV were more prominent in the right than left frontal lobe gyri; these differences may relate to differences in distributions of sex hormones.

According to current findings, there was significant negative correlation between age and GMV of the right, left, and total frontal lobes and the right superior frontal gyrus in males and females; the right pars orbitalis and orbitofrontal gyri in males; and the cingulate gyrus in females.

The results of the present study consistent with the voxelbased morphometric (VBM) studies found the reduction in GMV with age in superior and inferior frontal gyrus (Taki et al., 2011b), cingulate, and orbitofrontal gyri (Terribilli et al., 2011). In contrast, MRI studies using Statistical Parametric Mapping 2 (SPM2) on 1460 healthy subject aged 20-69 years, showed grater decline in GMV of precentral and middle frontal gyri (Taki et al., 2011b). Voxel based morphometric study on 223 aged between 8-79 years detected GMV loss in dorsolateral frontal cortex and precentral gyrus (Grieve et al., 2005). This discrepancy may possibly relate to age of subjects. In the compared study, the inclusion of children and adolescents on one side and elderly subjects on the other probably led to an overlapping of maturation and neurodegenerative processes, which would have been differentially influenced by hormonal effects across separate age ranges.

The reduction in the GMV observed in young adulthood is thought to reflect primarily the maturation changes (Taki et al., $2011 \mathrm{~b}$ ); and is not directly connected with decreases in specific brain function (Taki et al., 2011a). Brain maturation, consisting of both regressive cellular events, such as synaptic pruning, and progressive cellular events, such as myelination, occurs simultaneously in the brain during childhood, adolescence, and young adulthood; and this events result in decline of GMV (Taki et al., 2011b).

An interesting result, GMV of the left frontal lobe gyri didn't decline with age, whereas GMV of the right frontal lobe gyri decline by age, this divergence is possibly related to differences in the time course of the myelination and synaptic pruning maturational processes.

The effect of age on WMV was not homogenous; there was significant positive correlation between age and WMV of the left and total cingulate gyri in females and the right paracentral gyrus in males; but significant negative correlation between age and WMV of the total precentral gyri in males.

Results regarding effect of age on WMV were inconstant. Some studies showed that the WMV increases until the $5^{\text {th }}$ 
decade of life (Bartzokis et al., 2001; Taki et al., 2011a). In contrast, other studies showed that WMV remain relatively stable in adults until the age of 60-70 years and decline thereafter (Jernigan et al., 2001; Miller, 1980).

The increase in the WMV in the cingulate and paracentral gyri is likely associated with continued myelination and axonal growth (Webb et al., 2001) and possibly reflecting increasing the complexity in connectivity with functional and structural development (Pfefferbaum et al., 2013). An increase in myelination and/or interconnectivity could facilitate the synchronous integration of information across many regions (Gould et al., 1999). The speed of neural transmission depends on the structural properties of the connecting fibers, including axon diameter and the thickness of the insulating myelin sheath (Happe et al., 1998).

The decrease in WMV of precentral gyrus may result from microscopic structural changes, which include dilatation of perivascular spaces (Fazekas et al., 1993) and gliosis (Grafton et al., 1991). Regarding the fact that decrease in white matter volume not start until late in life, this finding regarding total and/ or cerebral white matter but not regional white matter volumes.

This study included only the young adult to clarify the impact of sex and age on grey matter volume (GMV) and white matter volume (WMV) of frontal lobes and their gyri. Sex has prominent effect on GMV and WMV of frontal lobe and most of frontal lobe gyri, with males had greater GMV and WMV of the frontal lobes and most of the frontal lobe gyri. Sex differences in GMV and WMV may have functional significant and/ or constitute sex-dependent redistributions of tissue volume. Further studies are important to find out the relationship between cognitive function and GMV and WMV.

Decrease in GMV by age has been reported in the right, left, and total frontal lobe; and right superior frontal, pars orbitalis, orbitofrontal gyri, and cingulate gyri. Increase in WMV by age has been reported in the left and total cingulate and right paracentral gyri. The findings of the present study demonstrate continuation of maturation of frontal lobe until third decade of age. These data are useful as baseline data for tracking diseases that cause progressive changes in GM and WM volumes.

\section{Conflict of interest}

None to declare.

\section{Acknowledgments}

This study was supported by Organization for Women in Science for Developing World (OWSD).

\section{References}

1. Allen, J. S., Damasio, H., Grabowski, T. J., Bruss, J., Zhang, W., 2003. Sexual dimorphism and asymmetries in the gray-white composition of the human cerebrum. Neuroimage. 18(4): 880-894.

2. Bartzokis, G., Beckson, M., Lu, P. H., Nuechterlein, K. H., Edwards, N., Mintz, J. Age-related changes in frontal and temporal lobe volumes in men: A magnetic resonance imaging study. Arch. Gen. Psychiatry. 58 (5), 461-465.

3. Brabera, A.D., Enta, D.V., Stoffers, D., Hansen, K.L., Boomsma, D., Geusa, E.D., 2013. Sex differences in gray and white matter structure in age-matched unrelated males and females and opposite-sex siblings. Int. J. Psychol. 6, 7-21.

4. Draganski, B., Gaser, C., Busch, V., Schuierer, G., Bogdahn, U., May, A., 2004. Neuroplasticity: Changes in grey matter induced by training. Nature. 427(6972), 311-312.

5. Elaine N., Marieb, K.H., 2007. Central Nervous System. Human Anatomy and Physiology. Pearson Education. Inc: Benjamin Cummings. p E.

6. Fazekas, F., Kleinert, R., Offenbacher, H., Schmidt, R., Kleinert, G., Payer, F., Radner, H., Lechner, H., 1993. Pathologic correlates of incidental MRI white matter signal hyperintensities. Neurology. 43(9), 1683-1689.

7. Gould, E., Reeves, A.J., Graziano, M.S., Gross, C.G., 1999. Neurogenesis in the neocortex of adult primates. Science. 286(5439), 548-552.

8. Grafton, S. T., Sumi, S. M., Stimac, G. K., Alvord, E. C., Jr., Shaw, C.M., Nochlin, D., 1991. Comparison of postmortem magnetic resonance imaging and neuropathologic findings in the cerebral white matter. Arch. Neurol. 48(3), 293-298.

9. Grieve, S.M., Clark, C.R., Williams, L.M., Peduto, A.J., Gordon, E., 2005. Preservation of limbic and paralimbic structures in aging. Hum. Brain Mapp. 25(4), 391-401.

10. Happe, F.G., Winner, E., Brownell, H., 1998.The getting of wisdom: theory of mind in old age. Dev. Psychol. 34(2), 358-362.

11. Jernigan, T.L., Archibald, S. L., Fennema-Notestine, C., Gamst, A.C., Stout, J.C., Bonner, J., Hesselink, J.R., 2001. Effects of age on tissues and regions of the cerebrum and cerebellum. Neurobiol. Aging. 22(4), 581-594.

12. Kasiri. K, Dehghani. M. J, Kazemi. K, Helfroush. M. S, Kafshgari. S., 2010. Comparison Evaluation of Three Brain MRI Segmentation Methods in Software Tools. Iranian Conference of Biomedical Engineering.

13. Kennedy, K.M., Erickson, K.I., Rodrigue, K.M., Voss, M.W., Colcombe, S.J., Kramer, A.F., Acker, J.D., Raz, N., 2009. Agerelated differences in regional brain volumes: A comparison of optimized voxel-based morphometry to manual volumetry. Neurobiol. Aging, 30(10), 1657-1676.

14. Martini, L., Melcangi, R. C., 1991. Androgen metabolism in the brain. J Steroid Biochem Mol Biol, 39(5B), 819-828.

15. Miller AK, A.R., Corsellis J.A., 1980. Variation with age in the volumes of grey and white matter in the cerebral hemispheres of man: measurements with an image analyser. Neuropathol. Appl. Neurobiol. 2, 119-132.

16. Paus, T., Toro, R., 2009. Could Sex Differences in White Matter be Explained by g ratio? Front. Neuroanat. 3, 14.

17. Pfefferbaum, A., Rohlfing, T., Rosenbloom, M.J., Chu, W., Colrain, I.M., Sullivan, E.V., 2013. Variation in longitudinal trajectories of regional brain volumes of healthy men and women (ages 10 to 85 years) measured with atlas-based parcellation of MRI. Neuroimage. 65, 176-193.

18. Schoenemann, P.T., Sheehan, M.J., Glotzer, L.D., 2005. Prefrontal white matter volume is disproportionately larger in humans than in other primates. Nat. Neurosci. 8(2), 242-252.

19. Snell, R.S., 2010. The Structure and Functional Localization of the Cerebral Cortex. Clinical Neuroanatomy. Philadelphia: Lippincott Williams \& Wilkins. 285-298. 
20. Taki, Y., Kinomura, S., Sato, K., Goto, R., Kawashima, R., Fukuda, H. A longitudinal study of gray matter volume decline with age and modifying factors. Neurobiol. Aging. 32(5): 907915, 2011a.

21. Taki, Y., Thyreau, B., Kinomura, S., Sato, K., Goto, R., Kawashima, R., Fukuda, H. Correlations among brain gray matter volumes, age, gender, and hemisphere in healthy individuals. PLoS One. 6(7), e22734, $2011 \mathrm{~b}$.

22. Terribilli, D., Schaufelberger, M.S., Duran, F.L., Zanetti, M.V., Curiati, P.K., Menezes, P.R., Scazufca, M., Amaro, E., Leite, C.C., Busatto, G.F. Age-related gray matter volume changes in the brain during non-elderly adulthood. Neurobiol. Aging. 32(2), 354-368, 2011.

23. Van Amelsvoort, T., Compton, J., Murphy, D., 2001. In vivo assessment of the effects of estrogen on human brain. Trends Endocrinol. Metab. 12(6), 273-276.
24. Webb, S.J., Monk, C.S., Nelson, C.A. Mechanisms of postnatal neurobiological development: implications for human development. Dev. Neuropsychol. 19(2): 147-171, 2001.

25. Welsh., M.C., Pennington, B.F. A normative developmental study of executive function: A window on prefrontal function in children. Dev. Neuropsychol. 7: 131-149, 1991.

26. Witte, A.V., Savli, M., Holik, A., Kasper, S., Lanzenberger, R. Regional sex differences in grey matter volume are associated with sex hormones in the young adult human brain. Neuroimage. 49(2): 1205-1212, 2010.

27. Woolley, C.S., Wenzel, H.J., Schwartzkroin, P.A. Estradiol increases the frequency of multiple synapse boutons in the hippocampal CA1 region of the adult female rat. J. Comp. Neurol. 373(1): 108-117, 1996. 